\title{
Hematopoietic stem cell transplantation for HIV cure
}

\author{
Daniel R. Kuritzkes
}

Division of Infectious Diseases, Brigham and Women's Hospital, Harvard Medical School, Boston, Massachusetts, USA.

\begin{abstract}
The apparent cure of an HIV-infected person following hematopoietic stem cell transplantation (HSCT) from an allogeneic donor homozygous for the ccr5 132 mutation has stimulated the search for strategies to eradicate HIV or to induce longterm remission without requiring ongoing antiretroviral therapy. A variety of approaches, including allogeneic HSCT from CCR5-deficient donors and autologous transplantation of genetically modified hematopoietic stem cells, are currently under investigation. This Review covers the experience with HSCT in HIV infection to date and provides a survey of ongoing work in the field. The challenges of developing HSCT for HIV cure in the context of safe, effective, and convenient once-daily antiretroviral therapy are also discussed.
\end{abstract}

\section{Introduction}

The advent of potent combination antiretroviral therapy (ART) has led to a dramatic decrease in the incidence of AIDS and AIDSrelated mortality worldwide. For most patients, full suppression of HIV-1 replication can be achieved by once-daily administration of an ART regimen available as a fixed-dose combination that is safe, convenient, and well tolerated. Five single-tablet regimens are currently approved by the US FDA for the initial treatment of HIV-1 infection, and several more are in phase III clinical trials (e.g., NCT02269917; NCT01797445; NCT02345226). Some studies suggest that, with early initiation of ART, patients with HIV-1 infection may live essentially normal life spans (1), converting what was once a uniformly fatal viral infection into a chronic disease that is manageable with appropriate medical treatment.

Although effective at restoring immune function and prolonging life, ART does not eliminate HIV-1, which persists as a latent infection in resting memory $\mathrm{CD}^{+} \mathrm{T}$ cells and possibly in cells of monocyte origin; treatment, therefore, must be administered throughout a patient's life. Despite the advances in HIV therapeutics of the last 30 years, concerns remain regarding the long-term safety of decades of ART, the burden of daily adherence, and the costs of providing lifelong ART on a global scale. In addition, higherthan-normal levels of immune activation persist in patients with full suppression of viral replication, and this activation is associated with an increased risk of end-organ disease, including myocardial infarction and stroke (2-4). A treatment that led to durable drugfree remission or eradication (cure) of HIV-1 could reduce the burden, cost, toxicities, and stigma associated with long-term ART and might lower immune activation and the associated risk of non-AIDS clinical events. The search for a cure therefore remains a high priority for clinicians, investigators, and patients.

As reviewed elsewhere in this issue (5), little or no transcription of proviral DNA occurs in resting $\mathrm{CD} 4^{+} \mathrm{T}$ cells that are latently infected with HIV-1. In the absence of any expression of viral pro-

Conflict of interest: D.R. Kuritzkes has received consulting honoraria from Bristol-Myers Squibb, Bionor, Gilead, InnaVirVax, Merck, and ViiV, as well as speaker fees from Gilead, Merck, and ViiV. He also has received research grant support from Gilead. Reference information: J Clin Invest. 2016;126(2):432-437. doi:10.1172/JCI80563. teins, these cells evade detection and destruction by the innate and adaptive immune systems. If latently infected cells could be eliminated (e.g., by ablative chemotherapy) and replaced through hematopoietic stem cell transplantation (HSCT) by uninfected cells or, ideally, cells intrinsically resistant to HIV-1 infection, cure might be achieved. This approach has attracted the interest of investigators for many years but, to date, has resulted in only a single successful outcome (6).

\section{Early experience with HSCT in HIV-infected patients}

The history of HSCT in HIV-infected patients has been reviewed by Hütter and Zaia (7). Early attempts to apply HSCT as an approach to immune reconstitution in patients with AIDS or as treatment for hematologic malignancies met with little success (8-10). Unsurprisingly, in the absence of effective ART, HSCT had little impact on the course of HIV disease and most patients died of progressive immunodeficiency or recurrent leukemia or lymphoma. In one case report, a patient with refractory lymphoma received an allogeneic HSCT from a matched, unrelated donor following conditioning with cyclophosphamide and totalbody irradiation (TBI), along with zidovudine, which had recently become available (11). Engraftment occurred on day 17, and the patient subsequently demonstrated complete chimerism. Virus was undetectable by culture or PCR of peripheral blood mononuclear cells (PBMC) beginning at day 32 after transplant. Unfortunately, the patient died of recurrent lymphoma at day 47, but HIV-1 was undetectable by culture or PCR of a variety of tissues obtained at autopsy. A similar outcome was reported in another patient who received an allogeneic HSCT and zidovudine; HIV-1 became undetectable by PCR in blood following engraftment, but the patient succumbed to graft-versus-host diseases (GVHD) (R. Saral and H.K. Holland, cited in ref. 7). In another case, a 25-year old woman with AIDS who received an allogeneic HSCT from a matched, unrelated donor after conditioning with busulfan and cyclophosmaide in the setting of ART with zidovudine and IFN- $\alpha 2$ survived for 10 months before succumbing to adult respiratory distress syndrome (12); tissues obtained at autopsy were negative for HIV-1 by PCR. 
By contrast, HIV remained readily detectable by culture or by PCR in patients who received HSCT from syngeneic donors. The largest such experience involved infusion of peripheral blood lymphocytes followed by BM transplantation in 16 HIV-infected patients; donors were HIV-uninfected identical twins (13). After transplantation, patients were randomized to receive zidovudine or placebo. Although the percentage of $\mathrm{CD}^{+} \mathrm{T}$ cells increased immediately after the infusions and transplantation, no clinical improvement was noted and patients remained viremic with positive cultures for HIV.

\section{HSCT in the HAART era}

The advent of highly active ART (HAART) in the mid-1990s transformed the treatment of HIV infection, resulting in a dramatic reduction in the incidence of AIDS and AIDS-related mortality (14). As a result, interest in the use of HSCT as a treatment for HIV infection waned, and attention shifted instead to its use for treatment of hematological malignancies and lymphomas in HIVinfected patients. Whereas earlier efforts were complicated by limited BM reserve and overall poor state of health of patients with advanced HIV disease, as well as synergistic toxicities due to the myelosuppressive effects of zidovudine $(15,16)$, the development of more potent and better tolerated ART regimens with fewer side effects allowed HIV-infected patients with leukemias or lymphomas to receive aggressive antineoplastic therapy, along with autologous or allogeneic HSCT (17-21). By the end of the first decade of the 2000s, the outcome of autologous HSCT in HIV-infected patients with lymphoma approached that in HIV-uninfected patients (22) and had become a standard of care (22-25).

Although clinical outcomes of HIV-infected patients with lymphomas have improved, there is little evidence that chemotherapy or HSCT have any sustained impact on the underlying HIV-1 infection. (A comprehensive discussion of the documented benefits of HSCT for HIV-infected patients with hematologic malignancies is beyond the scope of this review.) Low-level viremia in plasma and proviral DNA in PBMCs persists, despite autologous HSCT (26). Moreover, moderately intensive chemotherapy appears to have little lasting effect on HIV-1 persistence $(27,28)$. Possible explanations for this observation include the persistence of latently infected cells resistant to ablative chemotherapy and reinfusion of HIV-infected CD $4^{+} \mathrm{T}$ cells contaminating CD $34^{+}$stem cell preparations (26). In addition, due to concerns about pharmacological interactions between antiretroviral drugs and chemotherapeutic agents, it had become accepted practice to interrupt ART for a period of time during intensive chemotherapy or in the peritransplant period $(29,30)$. Consequently, even in the setting of allogeneic HSCT from an HIV-uninfected donor, donor-derived CD4 ${ }^{+}$ $\mathrm{T}$ cells would promptly become infected. Similar results were observed in macaques infected with a chimeric simian immunodeficiency virus/HIV (SHIV) and treated with suppressive ART followed by autologous HSCT (31). The monkeys also received pretransplant ablative chemotherapy and TBI that resulted in loss of $94 \%-99 \%$ of circulating $\mathrm{CD}^{+}{ }^{+} \mathrm{T}$ cells. Proviral SHIV DNA became undetectable after transplantation, but virologic rebound occurred rapidly in two of the animals following discontinuation of ART (a third animal had to be euthanized for clinical reasons prior to virologic rebound, but SHIV was detected by PCR in tis- sues obtained at necropsy). Thus, it appears that autologous HSCT alone is insufficient to eradicate HIV infection.

\section{Genetically modified hematopoietic stem cells for HIV cure}

As clinical outcomes in HIV-infected patients who received autologous HSCT improved, interest grew in genetically modifying stem cells to render them resistant to HIV-1 infection. Numerous approaches were explored, including the use of ribozymes to target HIV-1 or cellular genes, anti-sense RNAs, transdominant mutants, RNA decoys, siRNAs, and zinc-finger nucleases (32-35). Several of these approaches targeted expression of the C-C chemokine receptor 5 (CCR5) (36-38). Entry of HIV-1 into host target cells requires the binding of the envelope glycoprotein (gp120) to its primary receptor, CD4, followed by engagement of CCR5 or C-X-C chemokine receptor 4 (CXCR4) (39-44). Most HIV-1 isolates, known as R5 viruses, use CCR5 exclusively and cannot infect cells that do not express CCR5. Approximately $10 \%$ of the northern European population is heterozygous for a 32-bp deletion in CCR5 that renders the protein defective (45); the 1\% who are homozygous for this deletion have no detectable CCR5 on the surface of their $\mathrm{CD} 4^{+} \mathrm{T}$ cells and are resistant to infection with R5 HIV-1 (46). Blockade of CCR5 by small molecule antagonists such as maraviroc, approved for the treatment of HIV-1 infection, is well tolerated $(47,48)$. Therefore, disruption of CCR 5 by genetic modification of stem cells is likely to be safe.

Two newer approaches to gene editing include transcription activator-like effectors nucleases (TALENs) and engineered clustered regularly interspaced palindromic repeats (CRISPR) coupled to a CRISPR-associated (Cas) nuclease (e.g., Cas9) $(49,50)$. Like zinc-finger nucleases, TALENs use protein-mediated recognition of specific DNA sequences to direct the FokI nuclease to disrupt the targeted gene at the desired location. The CRISPR/Cas9 system accomplishes the same effect by use of a single-guide RNA to direct the Cas9 nuclease to the target gene. These approaches have been used in vitro to disrupt CCR5 expression in induced pluripotent stem cells, $\mathrm{T}$ cell lines, and primary $\mathrm{T}$ lymphocytes, rendering them resistant to HIV-1 infection (51-54).

Several logistical challenges have slowed the development of genetically modified hematopoietic stem cells as a potentially curative treatment for HIV infection. Chief among these challenges are concerns regarding the safety of HSCT in otherwise healthy HIV-infected patients on suppressive ART. Conventional wisdom suggests that some form of conditioning regimen is required to enhance engraftment of transduced stem cells. Although reduced-intensity conditioning regimens do not carry the same risks as fully myeloablative therapy, risk cannot be eliminated entirely and is greater than the risk of contemporary ART. In addition, there are concerns over insertional oncogenesis with approaches that rely on lentiviral transduction (55). For these reasons, initial clinical trials focused on HIV-infected patients who require allogeneic HSCT for treatment of hematologic malignancies (56). Moreover, since the transduced stem cells have no intrinsic selective advantage compared with untransduced host cells, interruption of ART may be required to allow HIV replication to eliminate unmodified, HIV-susceptible $\mathrm{CD}^{+} \mathrm{T}$ cells in order for the progeny of the modified stem cells to become pre- 
dominant. This problem is exacerbated by the generally low efficiency of transduction.

A potential solution to low transduction efficiency is to include a selectable marker in the vector, which allows enrichment of the gene-modified cells. One example of this approach is the incorporation of a truncated form of CD25 (tCD25) that does not bind IL-2 but can be recognized by anti-CD $25 \mathrm{mAb}$, allowing purification of successfully transduced cells by cell sorting (57). Even in this scenario, however, it is unclear how this approach would target and eliminate reservoirs of resting, latently infected CD4 ${ }^{+} \mathrm{T}$ cells and macrophages. Thus, despite keen interest in these approaches, significant hurdles remain.

\section{The Berlin patient}

An expedient alternative to generating CCR5 KOs is to perform HSCT using donors who are homozygous for the CCR5 432 deletion. Of course, this approach is limited to patients in need of HSCT for whom an adequate HLA match is available from a donor who happens to be CCR5-negative. Such was the case for a patient with acute myelogenous leukemia (AML) who received an HLA-matched HSCT from an unrelated donor homozygous for CCR5 32 (6). The patient, a 40 -year-old man living in Berlin, had been diagnosed with HIV-1 infection 10 years earlier and had been on a suppressive antiretroviral regimen including tenofovir (TDF), emtricitabine (FTC), and efavirenz (EFV) for four years prior to his AML diagnosis. The patient underwent two courses of induction chemotherapy and a single course of consolidation therapy, but his AML relapsed, necessitating HSCT. Due to the foresight of the patient's hematologist, an HLA-identical donor homozygous for the CCR5 432 allele was identified. The patient received anti-thymocyte globulin, cyclophosphamide, and TBI in preparation for the transplant; mycophenylate mofetil and cyclosporine were administered after HSCT to prevent GVHD. Although ART was interrupted at the time of HSCT, HIV-1 RNA remained undetectable in serum. Proviral HIV-1 DNA became undetectable in PBMCs after full chimerism was achieved on posttransplant day 61. Despite relapse of AML nearly one year later, HIV-1 RNA and DNA remained undetectable. The patient underwent a second HSCT from the same donor and has had sustained remission of AML and HIV-1 infection ever since. As of this writing, the patient has been free of detectable HIV-1 infection for approximately eight years without any ART. Extensive sampling of blood, rectal tissue, lymph node, cerebrospinal fluid, and brain has failed to yield confirmable evidence of persistent HIV-1 $(58,59)$. Thus, for all intents and purposes, this patient may be considered cured of HIV- 1 infection. Because of the impossibility of proving the complete absence of any persistent HIV-1 in this patient, the terms "functional cure" or "sustained remission" have also been applied.

\section{The Boston patients}

The apparent cure of the Berlin patient raised a number of important questions, including the relative contributions of the ablative conditioning regimen, posttransplant immunosuppressive therapy, GVHD, and receipt of donor cells lacking CCR5. We therefore examined the impact of allogeneic HSCT with WT donor cells on the HIV-1 reservoir in two HIV-1-infected patients with recurrent lymphoma (60). Both patients had previously undergone autolo- gous HSCT, which was unsuccessful in one case and led to myelodysplastic syndrome in the other. A unique aspect of these patients' care was that ART was continued throughout, with maintenance of HIV-1 suppression in the pre- and posttransplant period. Both patients also experienced clinically significant episodes of GVHD, which required treatment with corticosteroids and/or sirolimus and tacrolimus. A substantial reduction in proviral HIV-1 DNA in PBMCs occurred following full donor chimerism and recovery of $\mathrm{CD}^{+} \mathrm{T}$ cell counts. (Proviral DNA was also significantly reduced following HSCT in a third patient, but that patient died of recurrent malignancy before establishing full chimerism.) Significant reductions in HIV-specific antibody levels and avidity also were observed. Of note, both patients received a reduced-intensity conditioning regimen; neither patient received TBI or anti-thymocyte globulin. The loss of detectable HIV-1 after full donor chimerism strongly suggested that latently infected host cells were replaced by donor cells, which were protected from HIV-1 infection by the continued administration of ART. Because proviral HIV-1 DNA was readily detectable in PBMCs following administration of the conditioning regimen and became undetectable only with the establishment of full donor chimerism, it is tempting to conclude that GVHD played a significant role in reducing the peripheral viral reservoir by helping to clear infected host cells. Proviral DNA remained undetectable in both patients, even when larger numbers of $\mathrm{CD} 4^{+} \mathrm{T}$ cells harvested by leukapheresis were tested for HIV-1 DNA. No HIV-1 DNA was detectable in rectal mucosa of the one patient who agreed to a rectal biopsy, and attempts to recover infectious HIV-1 by in vitro stimulation of resting $\mathrm{CD} 4^{+} \mathrm{T}$ cells were unsuccessful. Neither patient had detectable cellular immune responses to HIV-1 antigens in vitro (61).

As a result of these findings, after extensive consultation with the patients' oncologists, infectious disease physician and the Institutional Review Board, both patients were offered the opportunity to interrupt ART with careful monitoring to determine whether HIV-1 had in fact been eradicated. At the time of ART interruption, the first patient was 2.5 years post-HSCT. When virus failed to rebound after approximately 2 months, treatment was interrupted in the second patient, who was 4.5 years postHSCT. Although both patients initially remained aviremic, plasma HIV-1 RNA rebounded after 12 weeks in the second patient and after 32 weeks in the first patient (61). In both cases, relapse was associated with symptoms and viral kinetics of primary HIV-1 infection. Symptoms resolved with reinitiation of ART, but a new EFV resistance mutation developed in one patient. Single-genome sequencing and phylogenetic analysis of Env from plasma virus at the time of relapse demonstrated that the virus was monophyletic and closely related to proviral HIV-1 DNA sequences present in PBMCs prior to HSCT, thereby excluding the possibility of reinfection and suggesting in both cases that relapse was initiated by activation of a single latently infected cell. Virus-specific cellular immune responses developed after HIV-1 rebound, as did new HIV-specific antibody responses (62).

While disappointing, these results yielded important insights into HIV-1 persistence and the challenges of viral eradication. The substantial reduction in the viral reservoir resulting from allogeneic HSCT allowed a variable period of ART-free remission of HIV-1 disease. Eventual viral rebound most likely occurred from long-lived 
Table 1. Ongoing and planned clinical trials of stem cell therapy for HIV-1 infection ${ }^{A}$

\begin{tabular}{|c|c|}
\hline Trial Number & Study Title \\
\hline NCT00569985 & $\begin{array}{l}\text { A Pilot Study of Safety and Feasibility of Stem Cell Therapy for Aids Lymphoma Using Stem } \\
\text { Cells Treated With a Lentivirus Vector Encoding Multiple Anti-HIV RNAs }\end{array}$ \\
\hline NCT00858793 & $\begin{array}{l}\text { High-dose Chemotherapy with Transplantation of Cene-modified Hematopoietic Stem Cells } \\
\text { for HIV-positive Patients with Malignant Diseases Indicating an HSCT }\end{array}$ \\
\hline NCT01213186 & $\begin{array}{l}\text { Phase II Study of Umbilical Cord-derived Mesenchymal Stem Cells in Restoring CD4+ T Cell } \\
\text { Counts and Reducing Immune Activation in HIV-infected Patients Underlying Long-term } \\
\text { Antiviral Therapy: a Multicenter, Does-escalating, Randomized, Double-blind, Controlled Trial }\end{array}$ \\
\hline NCT01734850 & $\begin{array}{l}\text { An Adaptive Phase I/II Study of the Safety of CD4 } 4^{+} \text {T Lymphocytes and CD34+ Hematopoietic } \\
\text { Stem/Progenitor Cells Transduced With LVsh5/C46, a Dual Anti-HIV Gene Transfer Construct, } \\
\text { With and Without Conditioning With Busulfan in HIV-1-Infected Adults Previously } \\
\text { Exposed to ART }\end{array}$ \\
\hline NCT01961063 & $\begin{array}{l}\text { Safety and Feasibility of Cene Transfer After Frontline Chemotherapy for Non-Hodgkin } \\
\text { Lymphoma in AIDS Patients Using Peripheral Blood Stem/Progenitor Cells Treated With a } \\
\text { Lentivirus Vector Encoding Multiple Anti-HIV RNAs }\end{array}$ \\
\hline NCT02140944 & $\begin{array}{l}\text { IMPAACT P1107: Cord Blood Transplantation With CCR5 } \triangle 32 \text { Donor Cells in HIV-1-Infected } \\
\text { Subjects Who Require Bone Marrow Transplantation for Any Indication and Its Observed } \\
\text { Effects on HIV-1 Persistence }\end{array}$ \\
\hline NCT02290041 & $\begin{array}{l}\text { Clinical Trial Phase I/II, of Test of Concept, Blind Double, Randomized, Controlled With } \\
\text { Placebo, to Assess the Safety and Efficiency of the Treatment With Allogenic Adult } \\
\text { Mesenchymal Stem Cells From Adipose Tissue Expanded, in HIV-infected Patients With } \\
\text { Controlled Viremia and Immunological Discordant Response }\end{array}$ \\
\hline NCT02337985 & $\begin{array}{l}\text { Safety and Feasibility of Stem Cell Gene Transfer Following R-EPOCH for Non-Hodgkin } \\
\text { Lymphoma in AIDS Patients Using Peripheral Blood Stem/Progenitor Cells Treated With } \\
\text { a Lentivirus Vector Encoding Multiple Anti-HIV RNAs }\end{array}$ \\
\hline NCT02343666 & $\begin{array}{l}\text { Clinical Trial of Gene-Modified Stem Cells to Generate HIV-Resistant Cells in Conjunction } \\
\text { With Standard Chemotherapy for Treatment of Lymphoma in Patients With HIV Infection }\end{array}$ \\
\hline NCT02378922 & $\begin{array}{l}\text { Autologous Transplantation and Stem Cell-Based Gene Therapy With LVsh5/C46 (CAL-1), } \\
\text { a Dual Anti-HIV Lentiviral Vector, for the Treatment of HIV-Associated Lymphoma }\end{array}$ \\
\hline NCT02500849 & $\begin{array}{l}\text { A Pilot Study to Evaluate the Feasibility, Safety and Engraftment of Zinc Finger Nuclease } \\
\text { (ZFN) CCR5 Modified CD34+ Hematopoietic Stem/Progenitor Cells (SB-728mR-HSPC) } \\
\text { in HIV-1 (R5) Infected Patients }\end{array}$ \\
\hline
\end{tabular}

Intervention
Transplantation of lentivirus vector rHIV7-shl-TAR-CCR5RZ-
transduced HSC
Transplantation of autologous CD34+ cells transduced with an
antiviral vector (M870).
Infusion (i.v.) of umbilical cord-derived mesenchymal
stem cells
Infusion of CD4+ T cells or CD34+ HSC transduced with LVsh5/
C46 (Cal-1)

Recombinant HIV7-shRNA targeted to the HIV-1 tat/rev-transactive response element-chemokine cysteine-cysteine receptor 5 ribozyme-treated hematopoietic stem progenitor cells

Transplantation with CCR5 $\triangle 32$ cord blood stem cells

Infusion (i.v.) of allogenic adult mesenchymal stem cells from adipose tissue

Infusion (i.v.) of lentivirus vector rHIV7-shl-TAR-CCR5RZtransduced hematopoietic stem/progenitor cells

Infusion (i.v.) of [46/CCR5/P140K lentiviral vector-transduced autologous HSC

Hematopoietic stem cell transplant with LVsh5/C46 (Cal-1) transduced CD34+ hematopoietic stem/progenitor cells

Infusion of ZFN CCR5 modified CD34+ hematopoietic stem/ progenitor cells (SB-728mR-HSPC)

AInformation gathered from www.clinicaltrials.gov (accessed 8/14/15). Excludes studies limited to lymphocyte infusion, stem cell transplantation for treatment of malignancy, and studies limited to use of genetically modified $\mathrm{CD} 4^{+} \mathrm{T}$ cells; completed or terminated studies are also excluded.

tissue reservoirs that persist at levels or in compartments in which remission is undetectable by current assays. The absence of detectable cellular immune responses and declining antibody titers during the period of ART-free remission suggest that virus-specific immunity did not play a significant role in limiting replication of these latent proviruses. This experience also demonstrates the challenge of proving that HIV-1 has been eradicated, despite the absence of detectable HIV-1 in blood and tissue samples, and underscores the importance of analytical treatment interruptions to assess the extent of HIV-1 reservoir depletion after therapeutic interventions.

\section{Other experience with allogeneic HSCT for HIV-1 cure}

Several additional reports on the effect of allogeneic HSCT on HIV-1 persistence have appeared since publication of the Berlin and Boston patients. Two patients in Sydney who received allogeneic HSCT for hematologic malignancies showed reductions in proviral DNA and HIV-1 antibody levels similar to that observed in the Boston patients (63). At last report, both patients remained on ART, so definitive conclusions regarding the extent of reservoir depletion cannot yet be drawn. Six other patients have received allogeneic HSCT from donors homozygous for CCR5432 (64). Unfortunately, all died within 12 months of HSCT from infection, GVHD, or recurrent lymphoma (five within two to four months). Three patients had received umbilical cord blood transplants, and three received HSCT from adult donors. Whether the use of donor cells lacking CCR5 contributed to the poor outcome in these six patients is difficult to assess.

One recipient of CCR5-negative donor stem cells in whom ART was interrupted at the time of myeloablative conditioning experienced HIV-1 relapse with CXCR4-tropic virus 20 days following HSCT (65). Retrospective analysis by deep sequencing identified a minority CXCR4-tropic variant in the provrial DNA from PBMCs obtained 103 days prior to HSCT that was identical to the virus that rebounded after HSCT; none of the pretransplant plasma virus sequences were strongly predictive of CXCR4 tropism. The patient died 373 days after HSCT of recurrent lymphoma, at which time CXCR4-tropic virus was again predominant in the plasma. This observation suggests that HIV eradication strategies dependent on interruption of CCR 5 expression may fail 
to control virus replication due to emergence of preexisting minority variants able to use CXCR4 for entry. There is no consensus at present regarding whether to screen potential recipients of HSCT from CCR5-negative donors for presence of minority CXCR4tropic virus populations.

\section{Conclusions}

The apparent cure of HIV-1 infection following allogeneic HSCT with cells from a $C C R 5^{-/-}$donor remains a singular event. Other attempts to replicate this result have failed thus far, but much has been learned about the viral reservoir and the relative contributions of conditioning regimens, GVH reaction, and coreceptor usage in establishing long-term, ART-free remission of HIV-1 infection. Although allogeneic transplantation of CCR $5{ }^{\mathrm{WT}}$ hematopoietic stem cells under the protection of continuous ART can lead to significant reductions in the viral reservoir, this approach fails to eradicate the virus. It appears that transplantation of stem cells intrinsically resistant to HIV-1 infection is necessary (but perhaps not sufficient) for this approach to succeed. Clearly, allogeneic HSCT is not a generalizable approach and is unlikely to be applied to healthy HIV-infected patients who would not otherwise be candidates for HSCT. Conversely, for patients with hematological malignancies who require HSCT, the priority should be to identify the best donor match to ensure success of the transplant and mini- mize the risk of GVHD; identifying a CCR5-negative donor should remain a secondary consideration at this time. In addition, every effort should be made to continue patients on ART throughout the conditioning phase and transplantation.

It is also clear that autologus HSCT of unmodified stem cells has minimal effects on the HIV-1 reservoir. Transplantation of autologous hematopoietic stem cells genetically engineered to resist HIV-1 infection is being tested in several pilot clinical trials (see Table 1). Whether or not this approach can, if safe and effective, be scaled up for delivery on a global scale, it is important that such experiments proceed in order to advance the field. Careful attention to appropriate patient selection, informed consent, and oversight by external monitoring committees is essential to safeguard the wellbeing of participants.

\section{Acknowledgments}

D.R. Kuritzkes was supported in part by U.S. Public Health Service grants UM1 AI068636, U01 AI117841, U01 AI114235, and R01 AI114617.

Address correspondence to: Daniel R. Kuritzkes, Division of Infectious Diseases, Brigham and Women's Hospital, 65 Landsdowne Street, Rm 449, Cambridge, Massachusetts 02139, USA. Phone: 617.767.8393; E-mail: dkuritzkes@partners.org.
1. van Sighem AI, Gras LA, Reiss P, Brinkman K, de Wolf F. Life expectancy of recently diagnosed asymptomatic HIV-infected patients approaches that of uninfected individuals. AIDS. 2010;24(10):1527-1535.

2. Kuller LH, et al. Inflammatory and coagulation biomarkers and mortality in patients with HIV infection. PLoS One. 2008;5(10):e203.

3. Tenorio AR, et al. Soluble markers of inflammation and coagulation but not T-cell activation predict non-AIDS-defining morbid events during suppressive antiretroviral treatment. J Infect Dis. 2014;210(8):1248-1259.

4. Hunt PW, et al. Gut epithelial barrier dysfunction and innate immune activation predict mortality in treated HIV infection. J Infect Dis. 2014;210(8):1228-1238.

5. Cary DC, Fujinaga K, Peterlin BM. Molecular mechanisms of HIV latency. J Clin Invest. doi:10.1172/JCI80565.

6. Hütter $\mathrm{G}$, et al. Long-term control of HIV by CCR5 $\Delta 32 / \Delta 32$ stem-cell transplantation. $N$ Engl JMed. 2009;360(7):692-698.

7. Hütter G, Zaia JA. Allogeneic haematopoietic stem cell transplantation in patients with human immunodeficiency virus: the experiences of more than 25 years. Clin Exp Immunol. 2011;163(3):284-295.

8. Hassett JM, Zaroulis CG, Greenberg ML, Siegal FP. Bone marrow transplantation in AIDS. N Engl JMed. 1983;309(11):665.

9. Verdonck LF, de Gast GC, Lange JM, Schuurman HJ, Dekker AW, Bast BJ. Syngeneic leukocytes together with suramin failed to improve immunodeficiency in a case of transfusion-associated AIDS after syngeneic bone marrow transplantation. Blood.1988;71(3):666-671.
10. Bardini G, Re MC, Rosti G, Belardinelli AR. HIV infection and bone-marrow transplantation. Lancet. 1991;337(8750):1163-1164.

11. Holland HK, et al. Allogeneic bone marrow transplantation, zidovudine, and human immunodeficiency virus type 1 (HIV-1) infection. Studies in a patient with non-Hodgkin lymphoma. Ann Intern Med.1989;111(12):973-981.

12. Contu L, et al. Allogeneic bone marrow transplantation combined with multiple anti-HIV-1 treatment in a case of AIDS. Bone Marrow Transplant. 1993;12(6):669-671.

13. Lane HC, et al. Syngeneic bone marrow transplantation and adoptive transfer of peripheral blood lymphocytes combined with zidovudine in human immunodeficiency virus (HIV) infection. Ann Intern Med. 1990;113(7):512-519.

14. Centers for Disease Control Prevention (CDC). Update: trends in AIDS incidence, deaths, and prevalence - United States, 1996. MMWR Morb Mortal Wkly Rep. 1997;46(8):165-173.

15. Kaplan LD, et al. Low-dose compared with standard-dose m-BACOD chemotherapy for non-Hodgkin's lymphoma associated with human immunodeficiency virus infection. National Institute of Allergy and Infectious Diseases AIDS Clinical Trials Group. N Engl J Med. 1997;336(23):1641-1648.

16. Levine AM, et al. Chemotherapy consisting of doxorubicin, bleomycin, vinblastine, and dacarbazine with granulocyte-colony-stimulating factor in HIV-infected patients with newly diagnosed Hodgkin's disease: a prospective, multi-institutional AIDS clinical trials group study (ACTG 149). J Acquir Immune Defic Syndr. 2000;24(5):444-450.

17. Ratner L, et al. Chemotherapy for human immunodeficiency virus-associated non-Hodgkin's lymphoma in combination with highly active antiretroviral therapy. J Clin Oncol. 2001;19(8):2171-2178.

18. Schneider E, et al. Autologous stem cell infusion for acute myeloblastic leukemia in an HIV-1 carrier. Bone Marrow Transplant. 1997;20(7):611-612.

19. Krishnan A, et al. Autologous stem cell transplantation for HIV-associated lymphoma. Blood. 2001;98(13):3857-3859.

20. Gupta V, et al. Allogeneic hematopoietic cell transplantation in human immunodeficiency virus-positive patients with hematologic disorders: a report from the center for international blood and marrow transplant research. Biol Blood Marrow Transplant. 2009;15(7):864-871.

21. Molina A, et al. High dose therapy and autologous stem cell transplantation for human immunodeficiency virus-associated non-Hodgkin lymphoma in the era of highly active antiretroviral therapy. Cancer. 2000;89(3):680-689.

22. Krishnan A, Palmer JM, Zaia JA, Tsai NC, Alvarnas J, Forman SJ. HIV status does not affect the outcome of autologous stem cell transplantation (ASCT) for non-Hodgkin lymphoma (NHL). Biol Blood Marrow Transplant. 2010;16(9):1302-1308.

23. Ambinder RF. The same but different: autologous hematopoietic stem cell transplantation for patients with lymphoma and HIV infection. Bone Marrow Transplant. 2009;44(1):1-5.

24. Krishnan A. Stem cell transplantation in HIV-infected patients. Curr Opin HIV AIDS. 2009;4(1):11-15.

25. Uldrick TS, Little RF. How I treat classical Hodgkin lymphoma in patients infected with human immunodeficiency virus. Blood. 2015;125(8):1226-1235. 
26. Cillo AR, et al. Plasma viremia and cellular HIV-1 DNA persist despite autologous hematopoietic stem cell transplantation for HIVrelated lymphoma. J Acquir Immune Defic Syndr. 2013;63(4):438-441.

27. Cillo AR, Krishnan S, McMahon DK, Mitsuyasu RT, Para MF, Mellors JW. Impact of chemotherapy for HIV-1 related lymphoma on residual viremia and cellular HIV-1 DNA in patients on suppressive antiretroviral therapy. PLoS One. 2014;9(3):e92118.

28. Delagrèverie HG, et al. Impact of combination of chemotherapy and autologous hematopoietic stem cell transplantation for lymphoma on HIV-1 reservoir persistance. Presented at: 22nd Conference on Retroviruses and Opportunistic Infections. International Antiviral Society-USA; February 23-26, 2015; Seattle, Washington, USA. Abstract 429.

29. Vishnu P, Aboulafia DM. AIDS-related nonHodgkin's lymphoma in the era of highly active antiretroviral therapy. Adv Hematol. 2012;2012:485943.

30. Miceli M, Cronin S, Ayash L, Alangaden G, Chandrasekar PH. Significant interaction of tacrolimus with ritonavir during allogeneic hematopoietic SCT in an HIV-infected patient. Bone Marrow Transplant. 2012;47(8):1140-1142.

31. Mavigner $\mathrm{M}$, et al. Persistence of virus reservoirs in ART-treated SHIV-infected rhesus macaques after autologous hematopoietic stem cell transplant. PLoS Pathog. 2014;10(9):e1004406.

32. Akkina R, Banerjea A, Bai J, Anderson J, Li MJ, Rossi J. siRNAs, ribozymes and RNA decoys in modeling stem cell-based gene therapy for HIV/ AIDS. Anticancer Res. 2003;23(3A):1997-2005.

33. Bonyhadi ML, et al. RevM10-expressing T cells derived in vivo from transduced human hematopoietic stem-progenitor cells inhibit human immunodeficiency virus replication. J Virol. 1997;71(6):4707-4716.

34. Tebas P, et al. Gene editing of CCR5 in autologous CD4 T cells of persons infected with HIV. N EnglJ Med. 2014;370(10):901-910.

35. Tebas P, et al. Antiviral effects of autologous CD4 $\mathrm{T}$ cells genetically modified with a conditionally replicating lentiviral vector expressing long antisense to HIV. Blood. 2013;121(9):1524-1533.

36. Shimizu S, et al. RNAi-mediated CCR5 knockdown provides HIV-1 resistance to memory $\mathrm{T}$ cells in humanized BLT mice. Mol Ther Nucleic Acids. 2015;4:e227.

37. Hofer U, Henley JE, Exline CM, Mulhern O, Lopez E, Cannon PM. Pre-clinical modeling of CCR5 knockout in human hematopoietic stem cells by zinc finger nucleases using humanized mice. J Infect Dis. 2013;208(suppl 2):S160-S164. 38. Li L, et al. Genomic editing of the HIV-1 core- ceptor CCR5 in adult hematopoietic stem and progenitor cells using zinc finger nucleases. Mol Ther. 2013;21(6):1259-1269.

39. Maddon PJ, Dalgleish AG, McDougal JS, Clapham PR, Weiss RA, Axel R. The T4 gene encodes the AIDS virus receptor and is expressed in the immune system and the brain. Cell. 1986;47(3):333-348.

40. Dalgleish AG, Beverley PC, Clapham PR, Crawford DH, Greaves MF, Weiss RA. The CD4 (T4) antigen is an essential component of the receptor for the AIDS retrovirus. Nature. 1984;312(5996):763-767.

41. Alkhatib G, et al. CC CKR5: a RANTES, MIP- $1 \alpha$, MIP-1 $\beta$ receptor as a fusion cofactor for macrophage-tropic HIV-1. Science. 1996;272(5270):1955-1958.

42. Choe S,et al. The beta-chemokine receptors CCR 3 and CCR 5 facilitate infection by primary HIV-1 isolates. Cell. 1996;85(7):1135-1148.

43. Deng HK, et al. Identification of a major coreceptor for primary isolates of HIV-1. Nature. 1996;381(6584):661-666.

44. Dragic T, et al. HIV-1 entry into $\mathrm{CD}^{+}$cells is mediated by the chemokine receptor CC-CKR- 5 . Nature. 1996;381(6584):667-673.

45. Martinson JJ, Chapman NH, Rees DC, Liu YT, Clegg JB. Global distribution of the CCR5 gene 32-basepair deletion. Nat Genet. 1997;16(1):100-103.

46. Samson M, et al. Resistance to HIV-1 infection in caucasian individuals bearing mutant alleles of th eCCR- 5 chemokine receptor gene. Nature. 1996;382(6593):722-725.

47. Gulick RM, et al. Maraviroc for previously treated patients with R5 HIV-1 infection. N Engl JMed. 2008;359(14):1429-1441.

48. Cooper DA, et al. Maraviroc versus efavirenz, both in combination with zidovudine-lamivudine, for the treatment of antiretroviral-naive subjects with CCR5-tropic HIV-1 infection. J Infect Dis. 2010;201(6):803-813.

49. Manjunath N, Yi G, Dang Y, Shankar P. New gene editing technologies toward HIV gene therapy. Viruses. 2013;5(11):2748-2766.

50. Hütter G, et al. CCR5 targeted cell terapy for HIV and prevention of viral escape. Viruses. 2015;7(8):4186-4203.

51. Mussolino C, Morbitzer R, Lutge F, Dannemann $\mathrm{N}$, Lahaye T, Cathomen T. A novel TALE nuclease scaffold enables high genome editing activity in combination with low toxicity. Nucleic Acids Res. 2011;39(21):9283-9293.

52. Choo SW, Kim S, Kim JM, Kim JS. Targeted genome engineering in human cells with the Cas9 RNA-guided endonuclease. Nat Biotechnol. 2013;31(3):230-232.
53. Lin Y, et al. Seamless modification of wildtype induced pluripotent stem cells to the natural CCR $5 \Delta 32$ mutation confers resistance to HIV infection. Proc Nat Acad Sci USA. 2014;111(26):9591-9596.

54. Mock U, et al. mRNA transfection of a novel TAL effector nuclease (TALEN) facilitates efficient knockout of HIV co-receptor CCR5. Nucleic Acids Res. 2015;43(11):5560-5571.

55. Cattoglio C, et al. Hot spots of retroviral integration in human $\mathrm{CD}_{3} 4^{+}$hematopoietic cells. Blood 2007;110(6):1770-1778.

56. DiGiusto DL, et al. RNA-based gene therapy for HIV with lentiviral vector-modified CD34(+) cells in patients undergoing transplantation for AIDS-related lymphoma. Sci Transl Med. 2010;2(36):36-43.

57. Barclay SL, et al. Safety and efficacy of a tCD 25 preselective combination anti-HIV lentiviral vector in human hematopoietic stem and progenitor cells. Stem Cells. 2015;33(3):870-879.

58. Allers K, et al. Evidence for the cure of HIV infection by CCR $532 / \Delta 32$ stem cell transplantation. Blood. 2011;117(10):2791-2799.

59. Yukl SA, et al. Challenges in detecting HIV persistence during potentially curative interventions: a study of the Berlin patient. PLoS Pathog. 2013;9(5):e1003347.

60. Henrich TJ, et al. Long-term reduction in peripheral blood HIV type 1 reservoirs following reducedintensity conditioning allogeneic stem cell transplantation. J Infect Dis. 2013;207(11):1694-1702.

61. Henrich TJ, et al. Antiretroviral-free HIV-1 remission and viral rebound after allogeneic stem cell transplantation: report of 2 cases. Ann Intern Med. 2014;161(5):319-327.

62. Stephenson KN, et al. HIV-1 reservoirs and humoral immunity in allogeneic stem cell transplantation patients. Presented at: 22nd Conference on Retroviruses and Opportunistic Infections, International Antiviral Society-USA; February 23-26, 2015; Seattle, Washington, USA. Abstract 430.

63. Koelsch KK, et al. Allogeneic bone marrow transplantation in two HIV-1 infected patients shows no detectable HIV-1 RNA or DNA, and a profound reduction in HIV-1 antibodies. Presented at: 20th International AIDS Conference; July 20-25, 2014; Melbourne, Australia. Poster LBPE21.

64. Hütter G. More on shift of HIV tropism in stemcell transplantation with CCR $532 / \Delta 32$ mutation. N Engl JMed. 2014;371(25):2437-2438.

65 . Verheyen J, et al. Breakthrough of preesixting X4-ccapable HIV after allogeneic stem-cell transplantation. Presented at: 22nd Conference on Retroviruses and Opportunistic Infections, International Antiviral Society-USA; February 23-26, 2015; Seattle, Washington, USA. Abstract 431. 